\title{
The Occurrence and Effects of Verbal and Visual Anchoring of Tropes on the Perceived Comprehensibility and Liking of TV Commercials
}

\section{Renske van Enschot \& Hans Hoeken}

To cite this article: Renske van Enschot \& Hans Hoeken (2015) The Occurrence and Effects of Verbal and Visual Anchoring of Tropes on the Perceived Comprehensibility and Liking of TV Commercials, Journal of Advertising, 44:1, 25-36, DOI: 10.1080/00913367.2014.933688

To link to this article: https://doi.org/10.1080/00913367.2014.933688

曲 Published online: 10 Nov 2014.

Submit your article to this journal $\sqsubset$

Џ Article views: 737

View Crossmark data $\complement$

4

Citing articles: 5 View citing articles $\square$ 


\title{
The Occurrence and Effects of Verbal and Visual Anchoring of Tropes on the Perceived Comprehensibility and Liking of TV Commercials
}

\author{
Renske van Enschot and Hans Hoeken \\ Radboud University, Nijmegen, the Netherlands
}

\begin{abstract}
For print advertising, it has been shown that rhetorical figures, tropes in particular, are used abundantly and that the effect on ad appreciation depends on the ad being perceived as comprehensible. To help the audience arrive at the intended interpretation, sometimes anchoring information is provided. The question is to what extent the same holds for TV commercials. A content analysis of $200 \mathrm{TV}$ commercials revealed that the vast majority contained tropes and (verbal and visual) anchoring information that either hinted at the intended interpretation or spelled it out completely; this anchoring information could be presented before/during the trope or after. For each commercial, aggregated consumer response data were analyzed. Verbally and visually spelling out the intended interpretation increased the perceived comprehensibility. For the visual anchoring information, this led to higher appreciation scores; for verbal anchoring information, increased comprehensibility canceled out the negative effect on appreciation caused by providing this information. Verbal anchoring information presented after the trope improved appreciation compared to it being presented before or during. Advertisers appear to take into account that solving the riddle posed by a trope may be difficult if the processing pace is set by the medium and provide additional help.
\end{abstract}

Rhetorical figures, more specifically tropes such as metaphors and puns, are omnipresent in advertising. Instead of simply stating that a new type of adhesive bandage is strong and protective, advertisers use a slogan such as "Say hello to your child's new bodyguard" (McQuarrie and Mick 1996). This

Address correspondence to Renske van Enschot, Radboud University, Nijmegen, Centre for Language Studies, P.O. Box 9103, 6500 HD, Nijmegen, the Netherlands. E-mail: r.vanenschot@let.ru.nl

Renske van Enschot (PhD, Radboud University, Nijmegen) is an assistant professor, Centre for Language Studies, Department of Dutch Language and Culture, Radboud University, Nijmegen.

Hans Hoeken ( $\mathrm{PhD}$, Tilburg University) is a professor, Centre for Language Studies, Department of Communication and Information Studies, Radboud University, Nijmegen.

Color versions of one or more of the figures in the article can be found online at www.tandfonline.com/ujoa. slogan poses a riddle the audience has to solve to arrive at the intended meaning. Studies have shown that over the years more and more print ads contain such figures, and that the number of figures in a single ad has increased as well (Ketelaar and van Gisbergen 2006; Phillips and McQuarrie 2002). Furthermore, there is ample evidence that rhetorical figures can influence ad liking because of the pleasure of processing they provide but only if the audience is able to come up with a solution to the riddle these rhetorical figures pose (e.g., Lee and Mason 1999; van Mulken, van Enschot-van Dijk, and Hoeken 2005). To help the audience to arrive at the intended meaning, the advertiser may provide a hint. Such hints, also known as anchoring, have been shown to increase the chances of understanding the trope, thereby increasing ad liking (e.g., Phillips 2000). However, the use of such anchoring information in print advertisements appears to be limited (Phillips and McQuarrie 2002; Ketelaar and van Gisbergen 2006).

The occurrence and impact of rhetorical figures have been studied mainly within the context of print advertising. However, Van Enschot, Beckers, and van Mulken (2010) show that the vast majority of television commercials contains tropes as well. Interpreting tropes in commercials may be more difficult because commercials contain more tropes as well as multimodal information. Even more important, where in print advertising the audience controls the speed with which it processes the ad, the processing pace is set by the commercial in television ads. This raises the question as to whether TV commercials contain more cues than print ads to facilitate the interpretation process and whether being able to come up with an interpretation also influences the liking of the commercial.

In this article, we focus on the occurrence and the impact of rhetorical figures in television commercials. First, 200 television commercials are analyzed with respect to the occurrence of tropes and the presence of anchoring information that may facilitate their interpretation. Second, it is assessed whether the attitude toward the commercial is influenced by the perceived comprehensibility of the commercial and whether that 
perceived comprehensibility depends on the presence of anchoring information.

\section{TROPES IN ADVERTISING}

The concept of rhetorical figures (e.g., Corbett and Connors 1999, McQuarrie and Mick 1996, 2003a) dates back to classic antiquity. They are stylistic devices functioning at the level of form instead of content. Rhetorical figures intend to communicate the message in an aesthetically pleasing way by deviating from the audience's expectations, thereby providing the audience with the "pleasure of text" (Barthes 1985; McQuarrie and Mick 2003a, p. 198, p. 208). Rhetorical figures can be classified into two main categories: schemes and tropes. Schemes deviate from the expectation that the distribution of sounds in an utterance is random. In ordinary language, words in a sentence seldom begin (alliteration) or end (rhyme) with the same sound. Copy writers make their slogans stand out by deviating from this expectation in slogans such as "Greyhound going great"; "Viakal: It's the Viakal fizz that does the bizz!"; and "Be wise, condomize." Schemes such as these do not alter the meaning of the message, nor do they require the audience to make inferences to get to the intended interpretation. This sets them apart from the other main category of rhetorical figures: tropes.

The defining feature of tropes (e.g., puns, metaphors) is that they violate our expectation that communicators aim to be perspicuous, that is, we expect that communicators try to avoid obscurity and ambiguity and be as brief and orderly as possible (Grice 1975). Tropes deviate from this expectation because there is more to them than meets the eye. A pun invites the audience to generate an additional meaning (e.g., General Electric's slogan "We bring good things to life"), a metaphor to conceive one thing in terms of another (e.g., "Say hello to your child's new bodyguards"). Tropes are found not only in the verbal but also in the visual part of utterances. Especially visual metaphors in print advertisements have been studied extensively (e.g., Forceville 1996; Phillips 2000; Phillips and McQuarrie 2004; van Mulken, le Pair, and Forceville 2010).

\section{PERCEIVED COMPREHENSIBILITY OF TROPES AND AD LIKING}

Being able to solve the riddle posed by the trope provides the audience with a pleasurable experience, which leads to a more positive attitude toward the ad (e.g., Ang and Lim 2006; Lee and Mason 1999; McQuarrie and Mick 2003b; Phillips 2000; van Mulken, van Enschot-Van Dijk, and Hoeken 2005; van Mulken, le Pair, and Forceville 2010). If people are unable to come up with a relevant interpretation for the trope, they get frustrated (e.g., van Enschot, Hoeken, and van Mulken 2008). Whether the interpretation is the one intended by the advertisers is not that important. Van Mulken, van Enschot-Van Dijk, and Hoeken (2005) showed that people who incorrectly believed that a slogan contained a pun evaluated the slogan more positively than those who correctly believed the same slogan did not contain a pun; conversely, people who incorrectly believed that a slogan did not contain a pun, appreciated the same slogan less than people who had identified the pun. These results show that subjectively experienced comprehension appears to be an important determinant for appreciation. This claim receives support from several studies reporting negative correlations between perceived comprehensibility and liking for tropes as diverse as irony (Burgers, van Mulken, and Schellens 2012), visual metaphors (van Mulken, le Pair, and Forceville 2010) as well as tropes in advertisements in general (Ketelaar et al. 2010; van Mulken, le Pair, and Forceville 2010).

To increase the chances of the audience coming up with the intended interpretation, the ad can contain anchoring. The concept of verbal anchoring dates back to Barthes (1977) who claimed that words can anchor implicit meanings, in his case, the meaning of press photos. Verbal anchoring consists of verbal elements that explain or help find a trope's meaning. Phillips (2000) studied the impact of verbal anchoring on the comprehension and interpretation in print advertising. One of the ads in her study was for a toothpaste brand that consisted of a picture of a pearl necklace positioned in such a way as to resemble smiling teeth. The intended interpretation was: This toothpaste will make your teeth pearly white. Apart from the version in which no verbal information was provided and the only cue to the intended interpretation consisted of the depiction of a toothpaste tube, Phillips constructed two versions including verbal anchoring. In one version, the verbal information hinted at the intended meaning ("Flash "em"), in the other version it was spelled out completely: "Makes your teeth pearly white." Phillips found that although comprehensibility peaked for the latter version, the version hinting at the intended meaning yielded the highest ad liking scores.

Phillips (2000) suggests that providing a hint to the intended meaning leads to higher ad liking scores compared to spelling out the intended meaning. The latter option would spoil the fun of solving the riddle. These results suggest that leaving the claim about the product's attribute too implicit may reduce the ad's likeability. However, Jeong (2008) did not find any differences on ad liking as a result of providing a hint to the intended meaning compared to providing no anchoring information at all, whereas Ketelaar and colleagues (2010) found that explicitly mentioning the intended meaning yielded more favorable attitudes toward the ad compared to a version containing no hints at all. The effect of anchoring information appears to run parallel to studies on the impact of explicit conclusions in advertising showing that people will only draw the implicit conclusion if they are highly involved (Kardes 1988; Sawyer and Howard 1991) or are very knowledgeable on the subject (Kardes, Kim, and Lim 1994). If people lack the motivation or ability, they may feel frustrated because they cannot figure out what the ad is intended to communicate. 
The studies discussed previously have all focused on the use of tropes in print advertising. Van Enschot, Beckers, and van Mulken (2010) show that tropes are a popular ingredient of television commercials as well. Approximately $82 \%$ of the commercials analyzed contained at least one trope. Anchoring these tropes may be even more important for television commercials as the pace at which the audience has to process the trope and come up with an interpretation is set by the medium instead of by the audience. This raises the question to what extent anchoring is used in television commercials and what the impact of anchoring is on ad liking.

\section{TROPES IN TELEVISION COMMERCIALS}

A trope can be found in a Volkswagen commercial (see Figure 1). We see a man moving on wheels, becoming smaller and overtaking other men on wheels. This commercial contains a trope. It artfully deviates from just communicating its claim straightforwardly by using a personification. The man stands for the sustainable Volkswagen TSI engine. The consumer needs to interpret the trope by replacing the man with the engine to arrive at the intended meaning: The TSI engine is smaller (needing less fuel) yet is still very powerful, enabling the driver to overtake other cars with larger engines.

In the Volkswagen ad, the intended interpretation is spelled out by a voiceover. There are commercials, however, in which no or only limited anchoring is provided and the audience has to work harder to arrive at the intended interpretation. In a commercial for the new Ford Mondeo, cars of other brands are shown as attached to balloons rising in the air, after which we see a Ford driving through the streets. The only (verbal) information provided is: "Mondeo. The new Ford." The audience has to infer that people want to get rid of their old cars because of the new Mondeo.

Ultimately, an ad's claim is of the type "product $\mathrm{X}$ has attribute Y" or "product X provides benefit Z" (Forceville 1996, p. 104; Maes and Schilperoord 2009). Commercials may depict one or both of these elements. That is, they may show the product and/or depict the attribute or benefit. In a commercial for a breakfast cereal containing chocolate, a piece of cereal is chasing bits of chocolate; after catching the chocolate, the cereal jumps back into the cereal box. The commercial visually depicts both the product (the cereal box as it can be bought in the supermarket) and the attribute (pieces of chocolate). In other commercials, the product is shown but the attribute is left implicit. For instance, in a commercial for Mazda, a shoal of fish is presented in which one fish takes a different direction. The car is shown but the benefit ("for those who want to go their own way") is left implicit. Studies on print advertising indicate that depiction of the product helps the audience arrive at the intended meaning (Dingena 1994; Ketelaar and van Gisbergen 2006). This suggests that providing visual information on the product and/or the attribute can be regarded as a form of visual anchoring.

In TV commercials, advertisers can choose not only to present verbal or visual cues to facilitate the comprehension process but also to control the moment these cues become available to the audience. That is, cues on how to interpret the trope can be provided before or during the trope is presented or after it has been presented. This difference in timing could

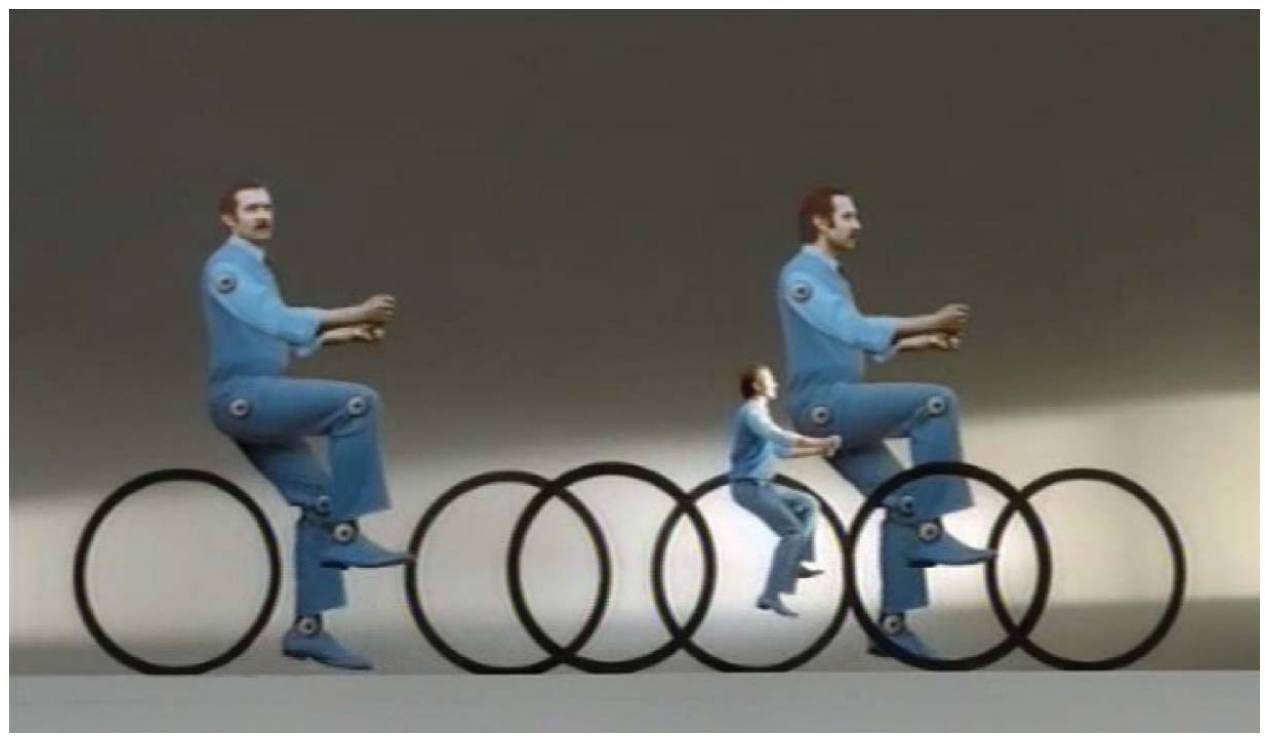

FIG. 1. Screenshot of Volkswagen commercial. Text of the Volkswagen commercial: "How does Volkswagen make cars more efficient and more sustainable? With the TSI engine, for instance. 'TSI' stands for 'Twin charged Stratified Injection,' That may sound complicated, but it simply boils down to the TSI engine being a lot smaller. As a result, it is much more fuel-efficient. And the beauty of it is: This is not at the expense of its power. In fact, the TSI engine is faster than many bigger engines. And it does so with only a little bit of energy. Less effort for the engine, so fuel-efficient. The sustainable Blue Motion technologies by Volkswagen." 
make a difference in the pleasure evoked by the trope. If the anchoring is presented before or during the presentation of the trope, the audience does not have to figure out the intended meaning by itself. If it is presented afterward, the audience has had the opportunity to solve the riddle after which it can check whether this interpretation was correct. The latter option enables the audience to experience the pleasure of text whereas the former option does not, or to a lesser extent. This raises the question as to whether the position of the anchoring influences the attitude toward the ad.

In this article, a study on the use, position, and impact of trope anchoring in television commercials is presented. In the first part, 200 commercials on a wide variety of products were analyzed to address the following research questions:

RQ1: To what extent do television commercials contain tropes?

RQ2: To what extent do television commercials containing tropes provide verbal and/or visual anchoring information?

RQ3: Is the anchoring information provided during or after the presentation of the trope?

In the second part, consumer response data for TV commercials are analyzed to assess to what extent verbal and visual anchoring of tropes influence the audience's perceived comprehensibility and attitude toward the commercial and to what extent this impact depends on the modality, the explicitness, and the position of the anchoring information.

\section{METHOD}

\section{Sampling Commercials}

A sample of 200 Dutch TV commercials was selected from a large set of approximately 450 commercials for which consumer response data had been gathered. All commercials had been broadcast on Dutch national TV during the period 2006 to 2010. From this original set, commercials were excluded for which relevant data were missing as well as commercials that were tested among very specific target groups. The remaining sample of 200 commercials represented a broad range of different brands and products, including fast-moving consumer goods, telecom, finance, electronics, energy, and automotive. Slightly more commercials (106) promoted products whereas the remaining 94 were about services.

\section{Procedure}

The analysis focused on the presence of tropes as well as on the extent of verbal and visual anchoring of these tropes. First, the 200 commercials were divided into two groups of $100 \mathrm{com}$ mercials. Second, each set of commercials was analyzed by two independent raters; thus, four raters were involved in this part. As a training session, the raters analyzed $20 \mathrm{TV}$ commercials before performing the actual content analysis. These commercials had been randomly selected and were not included in the actual content analysis. All raters examined the commercials carefully and several times before assigning a code.

The rating procedure consisted of four steps: First, the raters formulated the claim of a commercial (e.g., "This new deodorant contains natural ingredients" or "This insurance company reimburses more than other insurance companies") and resolved any disagreements in discussion. Second, they determined whether one or more rhetorical figures were present. Third, if a commercial contained a trope, the raters assessed the extent of verbal anchoring and/or visual anchoring (No/Incomplete/Complete). If the verbal part of the commercial communicated the claim of the commercial explicitly, this was classified as complete verbal anchoring. If the verbal part only hinted at the commercial's claim, this was classified as incomplete verbal anchoring. If both the product and the attribute were depicted, this was classified as complete visual anchoring; if either the product or the attribute was depicted, the commercial was classified as containing incomplete visual anchoring. Finally, the position of the anchoring information was determined (Before/During versus After the trope).

For the calculation of interrater reliability scores, one commercial per brand was selected if there were more commercials for the same brand, which resulted in 89 and 83 commercials respectively for the two groups of commercials. This was done to prevent that possible interdependent codings between commercials within one brand (using the same communication strategy) would inflate the interrater reliabilities results. Despite the fact that all raters had ample knowledge of rhetorical figures and experience in analyzing rhetorical figures in advertising, the initial interrater reliability scores were low with Cohen's kappas ranging from a minimum of .15 (for the extent of verbal anchoring as classified by the second pair of raters) to a maximum of .50 (for the position of the verbal anchoring).

Spooren and Degand (2010) argue that such low agreements between independent raters are inevitable given the complexity of the stimuli. To better exploit the raters' knowledge and experience and to increase the validity of the classification, the raters were independently presented with each other's classifications as well as the reasoning for these classifications in a second round. They were asked whether they would stick to their initial classification given the other rater's considerations or would like to change it. This second round resulted in much higher agreements between the two pairs of raters, ranging from .69 (extent of verbal anchoring) to .94 (for the extent of visual anchoring). Any remaining disagreements were resolved by the first author.

As a final check of the resulting classification, a fifth rater, who had not been involved in the previous rounds and who was naive to the study's research questions, was asked to indicate for a random selection of 100 commercials to what extent she considered the proposed claim an adequate representation 
of the commercial's message and whether the selected coding regarding trope presence was acceptable. If a commercial contained a trope, she indicated whether she agreed with the classification of the extent and location of verbal and visual anchoring. Without exception, the rater agreed with the interpretations and classifications generated by the pairs of raters.

\section{RESULTS}

Excluding all but one commercial for the same brand resulted in $153 \mathrm{TV}$ commercials being analyzed. ${ }^{1}$ The first research question was about the extent to which tropes were used in commercials. Over three-quarters of the commercials $(77.5 \%)$ contained a trope. Schemes were used in only $15.0 \%$ of the commercials. Almost three in ten (29.4\%) contained two or more rhetorical figures.

For the 119 commercials containing a trope, it was assessed to what extent they were verbally or visually anchored (research question 2; see Table 1). The vast majority (90: $75.6 \%$ ) contained complete verbal anchoring and a smaller set partial verbal anchoring (22: $18.5 \%)$, whereas very few commercials did not contain any verbal anchoring at all (7: 5.9\%). The absence of any visual anchoring was somewhat more common (22: $18.5 \%$ ), whereas the number of commercials containing partial visual anchoring (47: $39.5 \%)$ almost equaled the number of commercials containing complete visual anchoring (50: 42.0\%). Finally, for the position of the anchoring information (research question 3), a striking difference between verbal and visual anchoring was observed. Whereas verbal anchoring information was provided somewhat more often after the presentation of the trope than before or during (67 $(59.8 \%)$ versus $\left.45(41.2 \%), \chi^{2}(1)=4.32, p<.05\right)$, visual anchoring information was provided more often before or during (78: 80.4\%) the presentation of the trope instead of after (19: $\left.19.6 \% ; \chi^{2}(1)=35.89, p<.001\right)$.

TABLE 1

Extent of Anchoring $\times$ Location of Anchoring $(N)$

\begin{tabular}{lccc}
\hline & \multicolumn{2}{c}{ Location of anchoring } & \\
\cline { 2 - 3 } Extent of anchoring & Before/during & After & Total \\
\hline Verbal & & & \\
$\quad$ None & N/A & N/A & 7 \\
$\quad$ Incomplete & 5 & 17 & 22 \\
$\quad$ Complete & 40 & 50 & 90 \\
Visual & & & \\
$\quad$ None & N/A & N/A & 22 \\
Incomplete & 41 & 6 & 47 \\
Complete & 37 & 13 & 50 \\
\hline
\end{tabular}

Note. N/A indicates not applicable.

\section{DISCUSSION}

The results of the analysis show that similar to print advertising, the vast majority of TV commercials contain tropes. Whereas relatively few print ads with tropes provide anchoring information to help the audience arrive at the intended interpretation of the ad (Phillips and McQuarrie 2002; Ketelaar and van Gisbergen 2006), most TV commercials with tropes do provide verbal and/or visual anchoring information. Given that for TV commercials the pace at which the audience has to solve the riddle posed by the trope is set by the medium, providing additional cues to facilitate the interpretation process may be a wise choice. If the audience has difficulty coming up with a subjectively satisfying interpretation, this may result in a disliking of the commercial.

For all commercials in the sample, audience responses with regard to the commercials' perceived comprehensibility and appreciation have been gathered. These data enable to assess the impact of the extent of verbal and visual anchoring on the commercials' perceived comprehensibility and liking. Furthermore, compared to print ads, the position of the anchoring information can be varied. This raises the question of whether perceived comprehensibility and liking are influenced not only by the extent of anchoring but also by its position. Finally, given that both perceived comprehensibility and liking scores for each commercial have been obtained, it is possible to assess whether any effects on liking of the commercial are mediated through perceived comprehensibility. Therefore, the following research questions will be addressed in the second part of this study:

RQ4: To what extent do the extent and position of verbal anchoring influence the perceived comprehensibility and liking of the commercial?

RQ5: To what extent do the extent and position of visual anchoring influence the perceived comprehensibility and liking of the commercial?

RQ6: To what extent are any effects on the liking of the commercial mediated through perceived comprehensibility?

\section{METHOD}

\section{Data Collection}

For all 200 commercials in the sample, consumer response data were available. These data had been collected in 63 online surveys among on average 257 Dutch respondents per survey (ranging from 100 to 966). Respondents had been randomly selected from the MetrixLab multisource recruited Internet panel (consisting of approximately 600,000 panel members). Panel members are being recruited on over 500 different websites. The panel is carefully constructed and monitored to be representative with respect to sociodemographic variables such as gender, age, and education level. 
The response ratio of the surveys was between $20 \%$ and $25 \%$. Respondents may have been invited to participate in more than one survey but not in the same week and up to a maximum of 8 to 12 surveys a year (including surveys on different subjects, such as website usability and customer satisfaction). Respondents of the online surveys were paid $€ 1$ for their participation. The female:male ratio was on average 60:40. Education levels varied from lower vocational education to academic degrees, with largest groups at higher vocational education $(M=31.5 \%)$ and secondary vocational education and training $(M=25.7 \%)$. All respondents were over 16 years old; $53.2 \%$ of the respondents were 35 or younger, $35.3 \%$ were between 36 and 50, $11.6 \%$ were older than 50 .

Respondents were asked to watch the whole commercial and fill in their responses. Per survey, the responses to two to five $(M=4.4)$ commercials were registered. The following consumer responses were used in the present study: commercial likeability and perceived comprehensibility of the commercial. Both were measured on a single five-point semantic differential scale (Like versus Dislike and Easy to understand versus Difficult to understand, respectively). Per commercial, an average of consumers' scores was computed. This is consistent with the fact that managers' decisions about commercials are based on aggregated response scores (Stewart and Furse 2000). In this study, all commercials with tropes were used as a basis for the analysis (155). ${ }^{2}$ This includes commercials of the same brand, as each commercial of a brand was evaluated by a different respondent group.

\section{RESULTS}

ANOVA's were carried out to address research question 4 and research question 5. A summary of the results is provided in Table 2. First, a one-way ANOVA was conducted for the effect of extent of anchoring (No anchoring; Incomplete anchoring; Complete anchoring) on comprehensibility and liking scores separately. Verbal anchoring showed an effect for comprehensibility of the commercial $(F(2,152)=3.92, p<$ $.05, \eta^{2}=.049 ; M_{n o}=3.82, S D=0.48 ; M_{\text {incomplete }}=3.91$, $S D=0.42 ; M_{\text {complete }}=4.08, S D=0.34$ ), with a marginally

TABLE 2

Mean Scores of Commercial Comprehensibility and Likeability

\begin{tabular}{|c|c|c|c|c|c|c|}
\hline \multirow[b]{3}{*}{ Extent of anchoring } & \multicolumn{6}{|c|}{ Location of anchoring } \\
\hline & \multicolumn{2}{|c|}{ Before/during } & \multicolumn{2}{|c|}{ After } & \multicolumn{2}{|c|}{ Total } \\
\hline & $M(S D)$ & $n$ & $M(S D)$ & $n$ & $M(S D)$ & $n$ \\
\hline \multicolumn{7}{|c|}{ Verbal } \\
\hline \multicolumn{7}{|l|}{ Comprehensibility } \\
\hline None & N/A & N/A & N/A & N/A & $3.82^{\mathrm{a}}(0.48)$ & 7 \\
\hline Incomplete & $3.86(0.55)$ & 6 & $3.92(0.39)$ & 22 & $3.91^{\mathrm{a} \sim}(0.42)$ & 28 \\
\hline Complete & $4.07(0.37)$ & 57 & $4.08(0.31)$ & 63 & $4.08^{\mathrm{a} \sim}(0.34)$ & 120 \\
\hline Total & $4.05^{\mathrm{a}}(0.39)$ & 63 & $4.04^{\mathrm{a}}(0.34)$ & 85 & & \\
\hline \multicolumn{7}{|l|}{ Likeability } \\
\hline None & N/A & N/A & N/A & N/A & $2.99^{\mathrm{a}}(0.48)$ & 7 \\
\hline Incomplete & $3.01(0.55)$ & 6 & $3.45(0.53)$ & 22 & $3.35^{\mathrm{a}}(0.55)$ & 28 \\
\hline Complete & $3.09(0.50)$ & 57 & $3.35(0.51)$ & 63 & $3.23^{\mathrm{a}}(0.52)$ & 120 \\
\hline Total & $3.08^{\mathrm{a}}(0.50)$ & 63 & $3.38^{\mathrm{b}}(0.51)$ & 85 & & \\
\hline \multicolumn{7}{|c|}{ Visual } \\
\hline \multicolumn{7}{|l|}{ Comprehensibility } \\
\hline None & N/A & N/A & N/A & N/A & $3.92^{\mathrm{a}}(0.43)$ & 27 \\
\hline Incomplete & $3.93(0.36)$ & 50 & $3.94(0.35)$ & 8 & $3.93^{\mathrm{a}}(0.36)$ & 58 \\
\hline Complete & $4.17(0.34)$ & 47 & $4.15(0.26)$ & 23 & $4.16^{\mathrm{b}}(0.31)$ & 70 \\
\hline Total & $4.04^{\mathrm{a}}(0.37)$ & 97 & $4.10^{\mathrm{a}}(0.29)$ & 31 & & \\
\hline \multicolumn{7}{|l|}{ Likeability } \\
\hline None & N/A & N/A & N/A & N/A & $3.00^{\mathrm{a}}(0.62)$ & 27 \\
\hline Incomplete & $3.20(0.49)$ & 50 & $3.03(0.44)$ & 8 & $3.18^{\mathrm{a}}(0.48)$ & 58 \\
\hline Complete & $3.41(0.46)$ & 47 & $3.33(0.51)$ & 23 & $3.38^{\mathrm{b}}(0.47)$ & 70 \\
\hline Total & $3.30^{\mathrm{a}}(0.48)$ & 97 & $3.25^{\mathrm{a}}(0.50)$ & 31 & & \\
\hline
\end{tabular}

Note. The $n$ s refer to the number of commercials. Means: $1=$ Difficult to understand/dislike; $5=$ Easy to understand/like. Different superscripts indicate significant differences at $p<.05 ; \sim$ indicates a significant difference at $p<.10$; N/A indicates not applicable. 
significant difference between complete and incomplete verbal anchoring. ${ }^{3}$ Verbal anchoring had no impact on the likeability of the commercial $\left(F(2,152)=1.50, p=.23 ; M_{n o}=2.99\right.$, $S D=0.48 ; M_{\text {incomplete }}=3.35, S D=0.55 ; M_{\text {complete }}=3.23$, $S D=0.52)$. Regarding visual anchoring, commercials with complete visual anchoring $\left(M_{\text {complete }}=4.16, S D=0.31\right)$ were perceived as more comprehensible than commercials with incomplete visual anchoring $\left(M_{\text {incomplete }}=3.93, S D=0.36\right)$ and without visual anchoring $\left(M_{n o}=3.92, S D=0.43 ; F(2\right.$, $\left.152)=8.60, p<.001, \eta^{2}=.102\right)$. A similar effect was found for the likeability scores with complete visual anchoring yielding higher liking scores $(M=3.38, S D=0.47)$ than incomplete visual anchoring $(M=3.18, S D=0.48)$ and no visual anchoring $(M=3.00, S D=0.62 ; F(2,152)=6.30, p<.01$, $\left.\eta^{2}=.077\right)$.

Commercials with no anchoring information are not relevant for the analysis of the effect of position of anchoring information. Therefore, two separate 2 (Anchoring: Complete, Incomplete) $\times 2$ (Position: Before/During, After) univariate ANOVAs were carried out (research question 4 and research question 5). A summary of the results is provided in Table 2.

Commercials containing complete verbal anchoring were perceived as more comprehensible $(M=4.08, S D=0.34)$ than those containing incomplete verbal anchoring $(M=3.91$, $\left.S D=0.42 ; F(1,144)=4.48, p<.05, \eta^{2}=.030\right)$. However, there was no effect of the extent of verbal anchoring on the liking of the commercial $(F(1,144)<1)$. The opposite pattern was found for the location of anchoring. Whereas location had no effect on the perceived comprehensibility $(F(1,144)<1)$, commercials that presented the anchoring after the trope were liked better $(M=3.38, S D=0.51)$ compared to those presenting the anchoring before or during the trope $(M=3.08, S D=$ $\left.0.50 ; F(1,144)=7.68, p<.01, \eta^{2}=.051\right)$. The two factors did not interact for any of the dependent variables $(F \mathrm{~s}(1,144)$ $<1)$.

With respect to visual anchoring, Location had no impact on the perceived comprehensibility $(F(1,124)<1)$ and likeability $(F(1,124)=1.32, p=.25)$ of the commercials. Furthermore, location did not interact with the extent of visual anchoring $(F \mathrm{~s}(124)<1)$. However, commercials containing complete visual anchoring were perceived as more comprehensible $(M=$ $4.16, S D=0.31)$ than those with incomplete visual anchoring $\left(M=3.93, S D=0.36 ; F(1,124)=8.67, p<.01, \eta^{2}=.065\right)$. A similar effect was obtained for the liking scores with complete visual anchoring yielding higher liking scores $(M=3.38$, $S D=0.47)$ than incomplete visual anchoring $(M=3.18, S D=$ $\left.0.48 ; F(1,124)=5.33, p<.05, \eta^{2}=.041\right)$.

To explore the potential mediating role of perceived comprehensibility (research question 6), mediation analyses were conducted for the impact of verbal and visual anchoring separately (Preacher and Hayes 2008). We used Preacher and Hayes's (2008) bootstrapping method for testing indirect effects. The bootstrap data can be interpreted by determining whether the confidence interval of the indirect effect (i.e., paths $a$ and $b$ combined; see the appendix) contains zero. If this interval does not contain zero, a significant mediation (or suppression) effect occurs.

With respect to the extent of verbal anchoring, the ANOVAs did not reveal any differences in likeability of the commercial (this is reflected in the insignificant total effect (path c); see Figure A1. However, the mediation analyses disclosed that commercials with incomplete anchoring were essentially better liked (see the direct in effect in Figure A1) but that this effect was suppressed by their lower perceived comprehensibility scores (random indirect effect estimate: $-.13, \mathrm{SE}=.05,95 \% \mathrm{CI}=-.03,-.24$; see paths $\mathrm{a}, \mathrm{b}$, and $\mathrm{c}^{\prime}$ in Figure A1). ${ }^{4}$ In other words, complete verbal anchoring yielded higher perceived comprehensibility scores, which in turn had a positive effect on the appreciation of the commercial, thereby suppressing the direct negative effect a complete spelling out of the message had on appreciation.

With respect to visual anchoring, the extent of anchoring had a significant effect on both perceived comprehensibility and likeability (this is, for instance, reflected in the significant total effect of extent of visual anchoring on commercial likeability; see Figure A2). A mediation analysis revealed that the effect on appreciation was fully mediated by perceived comprehensibility (random indirect effect estimate: $-.13, \mathrm{SE}=$ $.04,95 \% \mathrm{CI}=-.06,-.22$; see paths $\mathrm{a}, \mathrm{b}$, and $\mathrm{c}^{\prime}$ in Figure A2). In other words, the positive effect of complete versus incomplete visual anchoring on appreciation was caused by the complete visual anchoring yielding higher perceived comprehensibility scores, which in turn led to higher appreciation scores.

\section{DISCUSSION}

The results show that perceived comprehension plays an important role with respect to the appreciation of TV commercials. With respect to verbal anchoring, complete anchoring boosts the perceived comprehension of the commercial thereby suppressing the negative impact on appreciation that verbally spelling out the intended message has. Complete visual anchoring also increases the perceived comprehensibility. Compared to complete verbal anchoring, however, people are not bothered by a completely spelled out message in the visual mode. Therefore this higher perceived comprehensibility yields a higher appreciation for the commercial as well. Finally, the timing of the verbal anchoring is important too. When the anchoring followed the trope, the commercial was better liked than when it was presented before or during the presentation of the trope.

\section{GENERAL DISCUSSION}

This study shows that the abundant use of tropes is not restricted to print advertisements; the vast majority of Dutch 
TV commercials contain them as well. In his meta-analysis on the impact of humor in advertising, Eisend (2011) showed that the positive feelings evoked by an ad are translated, admittedly to a lesser extent, into a more favorable brand attitude. Given that the use of tropes in advertising can evoke such feelings, it appears plausible that the use of tropes is not restricted to print advertising but that the use of tropes is a popular message strategy in TV commercials as well.

Research on print advertising as discussed in the introduction has shown that for tropes to evoke favorable feelings they need to be perceived as comprehensible by the audience (e.g., Lee and Mason 1999; Phillips 2000; van Enschot, Hoeken, and van Mulken 2008; van Mulken, van Enschot-Van Dijk, and Hoeken 2005). Content analyses have shown that print ads with tropes nevertheless contain few cues that may help the audience to get to the intended interpretation (e.g., Phillips and McQuarrie 2002). In this study, however, only 3 out of 119 TV commercials with tropes did not contain any anchoring at all, whereas the vast majority presented the audience either verbally or visually with the intended interpretation. It appears that advertisers take into account that solving the riddle posed by a trope may be more difficult if the pace of processing is set by the (audiovisual) medium and additional help or even providing the solution is necessary.

The results provide a different picture with respect to the impact of the degree and the location of verbal anchoring compared to that of the degree and the location of visual anchoring. This difference is in accordance with the way in which tropes can lead to a higher appreciation of the commercial. Tropes can be used to communicate the intended meaning (e.g., product $\mathrm{X}$ has attribute $\mathrm{Y}$; product $\mathrm{X}$ provides benefit $\mathrm{Z}$ ) in an indirect way. Hoeken and colleagues (2009) argue that when an advertiser spells out this intended meaning, the advertiser sends the message to the audience that he or she believes the audience to be unable to come up with the intended interpretation on its own. As a result, "the audience may feel patronized," and this feeling is likely to result in a more negative appreciation of the message (Hoeken et al. 2009). However, the findings of this study suggest that this negative effect occurs only for complete verbal anchoring information (and not for complete visual anchoring information) and when it is presented before or during the presentation of the trope (and not if it is presented afterward).

The difference in effect of complete verbal versus complete visual anchoring can be the result of the fact that processing the verbal anchoring information immediately yields the intended interpretation (e.g., "product $\mathrm{X}$ has attribute $\mathrm{Y}$ "), whereas the visual anchoring information still requires additional inferences to arrive at the intended interpretation. Sperber and Wilson (1995, p. 175) state that all nonverbal communication is "weak communication," in the sense that one can never be sure which message the sender is aiming to convey. Messaris (1997, p. xix) makes a similar claim when discussing the use of images in advertising. Complete visual anchoring information requires the audience to make additional inferences about the intended meaning of the ad, whereas complete verbal anchoring information does not. Verbal anchoring information may have given the audience the impression that the advertiser doubted its ability to arrive at the intended meaning, which in turn may have led to more negative appreciation scores.

Presenting the verbal anchoring information before or during the presentation of the trope also had a negative impact on the appreciation of the commercial. This situation may resemble the situation in which someone asks you a question and provides the answer before you had the chance to come up with the answer yourself. As for the effect of complete verbal anchoring information, this timing of presenting anchoring information may have given the impression that the advertiser thought that the audience would not be able to come up with the solution to the riddle itself. Providing the verbal anchoring information after the presentation of the trope may have diminished the feeling of being patronized. The fact that the position of the visual anchoring information had no impact on appreciation is in line with the explanation provided in the previous paragraph; visual anchoring information always requires an additional inference to generate the intended meaning.

The responses of the audience attest to the importance of the perceived comprehensibility for the appreciation of the commercial. As expected, spelling out the intended interpretation of a trope, whether verbally or visually, increased the perceived comprehensibility of the commercial. In both cases, this had a positive effect on the liking of the commercial, although in slightly different ways. In the visual mode, the higher perceived comprehensibility scores yielded higher appreciation scores directly, whereas in the verbal mode they canceled out the negative effect of spelling out the intended meaning on appreciation. This pattern of results is highly similar to the one obtained by Burgers, van Mulken, and Schellens (2012) for the impact of verbal irony. In that study, a negative effect of irony on perceived comprehensibility suppressed the positive effect of irony on appreciation.

\section{LIMITATIONS AND FUTURE RESEARCH}

In this study, professional commercials that have been aired in the Netherlands were analyzed. Obviously, these commercials differed on many dimensions beyond the presence of tropes and their anchoring. As a result, it is impossible to rule out that other characteristics co-occurring with tropes and anchoring are responsible for the effects obtained. However, the fact that the presence of (complete) verbal and visual anchoring influenced the perceived comprehensibility, and the fact that mediation analyses proved these scores to be responsible for the appreciation scores, lends the results at least face validity. Nevertheless, experimental research is in order in which the presence of tropes and the extent and position of anchoring is carefully manipulated. Such experiments could 
also measure the degree to which participants feel patronized by the advertiser when the verbal anchoring information completely spells out the intended interpretation or when this information presented before or during the presentation of the tropes. Including this measure would enable to assess whether the negative effects of verbal anchoring information on appreciation is mediated by the feeling of being patronized.

The two main dependent variables in this study, perceived comprehensibility and appreciation of the commercial, were each measured using a single item. From a reliability perspective, single-item measurements are less desirable. However, the scores for the commercials were based on the average ratings of large numbers of participants who varied quite strongly with respect to age, gender, and level of education. As the analyses were conducted on the average scores for these heterogeneous samples, the perceived comprehensibility and appreciation scores can be considered reliable. When conducting experiments on the impact of tropes in commercials, it would be advisable to employ validated multiple items scales to measure perceived comprehensibility and the appreciation of the ad.

Apart from using such self-report scales after participants have viewed the commercials, it would be interesting to apply think-aloud measures. Having participants express their thoughts while watching the commercials not only may provide insights into the depth of and style of processing but also can show how the completeness and the position of the verbal anchoring information influences the comprehension and appreciation of the commercial. Having participants express their thoughts when watching the commercial could enable a test of the explanation that people feel being patronized by completely spelled out verbal anchoring information or by provided information before they had a chance to come up with the solution themselves.

All commercials were aired on Dutch television and the ratings were provided by Dutch participants. This raises the question to what extent the pattern of results reported in this article applies only to the Dutch situation. Studies comparing different cultures provide no reason for alarm in this respect as long as the comparison concerns Western cultures with developed markets. Ketelaar and Van Gisbergen (2006) reported similar results for the use of tropes and of verbal anchoring information in the Netherlands as had been reported for the United States by Phillips and McQuarrie (2002). Ketelaar and colleagues (2010) also report that Dutch participants respond in a similar way to tropes as the U.S. participants did in the study by Phillips (2000). In a number of studies conducted within a European context, no differences were found in responding to different advertising appeals when comparing Dutch, Belgian, English, French, German, and Spanish participants (Hoeken et al. 2003; Hoeken et al. 2007). Finally, van Mulken and her colleagues presented ads containing tropes to Dutch, French, German, and Spanish participants and did not find cultural differences in responses to these ads (van Hooft, van Mulken, and Nederstigt 2011; van Mulken, le Pair, and Forceville 2010).
The studies discussed previously have all been conducted in Western cultures with developed markets. The question is whether differences can be expected when comparisons are made between Western and Eastern cultures or between emerging and developed markets. There is ample research showing important differences in the systems of thoughts between Eastern and Western cultures (Nisbett 2003). These differences may have consequences for the processing and appreciation of advertising in different cultures, and there is indeed evidence for such different consequences. Hornikx and O'Keefe (2009) conducted a meta-analysis on the question of whether there are cultural differences in the impact of advertising. The only cultural difference they were able to find was between Western, more individualistic cultures and Eastern, more collectivistic cultures. Zhou and Belk (2004), employing a reader-response approach, documented the specific ways in which Chinese consumers interpret (global) ads and commercials. With respect to the emerging versus developed markets divide, Zarantonello, Jedidi, and Schmitt (2013) show that whereas advertising in developed markets is characterized by its reliance on evoking sensations, feelings, and imaginations, advertising in emerging markets is more characterized by emphasizing the features and benefits of the products. The use and impact of rhetorical figures in developed markets may therefore differ from those in emerging markets.

Finally, the category of tropes is a broad one and consists of, for example, metaphors, puns, hyperboles, and irony (e.g., Corbett and Connors 1999; McQuarrie and Mick 1996; Phillips and McQuarrie 2002). This study focused on the common denominator of tropes and not on their differences. Tropes were therefore not subdivided. Nevertheless, some tropes may be easier to understand than others and may not need to be anchored. Le Pair and van Mulken (2010) performed a study in which they anchored more or less complex visual metaphors in print advertising. The most complex visual metaphor yielded the strongest effect of verbal anchoring on experienced complexity. The reduced complexity did not make the advertisement more attractive. The other, less complex metaphors showed weaker effects or no effects at all. Future research can build on this study using other kinds of tropes differing in complexity, focusing not only on verbal anchoring but on visual anchoring as well, and looking at TV instead of print.

\section{MANAGERIAL IMPLICATIONS}

Advertisers are confronted with the problem that they have to find new ways to communicate the same message (e.g., our beer is refreshing, our toothpaste makes your teeth white). If they fail to do so, the audience will ignore the advertisement if it has seen the ad too often. Advertisers therefore take their refuge to using rhetorical figures such as tropes to present their message in a new way, and they do so not only in print advertisements but in TV commercials as well. In this article, we have shown that tropes in TV commercials can lead to more liking of these commercials if 
the commercial contains information that ensures that the audience will get the intended meaning. This information can be provided in a verbal or visual format. If verbal anchoring is employed, it appears advisable to present this information after the trope has been presented.

\section{NOTES}

1. Given that 89 commercials for different brands were analyzed by the first pair of raters and 83 commercials for different brands by the second pair, one might expect the number of commercials to be $(89+83=) 172$ commercials instead of 153 . However, among the 172 commercials, there were 19 brands for which 2 commercials had been analyzed: one by the first pair of raters, the other by the second pair of raters. The criterion that for each brand only one commercial was entered into the main analysis led to the random selection of one of the two commercials. This resulted in a sample of 153 commercials instead of 172 .

2. When addressing research questions 4,5 , and 6 , the data for all commercials containing rhetorical figures were taken into account, based on the original set of 200 commercials. There were $155 \mathrm{com}$ mercials containing rhetorical figures. For each of these commercials, the presence of rhetorical figures and the extent and position of anchoring information was assessed by two independent raters.

3. Only the comparison between the complete and the incomplete verbally anchored commercials approached significance, whereas that was not the case for the comparisons with the commercials without verbal anchoring information. This is probably the result of the low number of commercials in the latter condition.

4. Three conditions should be satisfied for suppression to occur: (1) the extent of anchoring should influence commercial comprehensibility (effect a), (2) commercial comprehensibility should influence commercial likeability (effect b; see Preacher and Hayes 2008), and (3) the polarity of ab should be different from $\mathrm{c}^{\prime}$ (Shrout and Bolger 2002). The third condition implies that if ab yields a positive operator, $\mathrm{c}^{\prime}$ should yield a negative operator and vice versa. In other words, complete verbal anchoring yielded higher perceived comprehensibility scores, which in turn had a positive effect on the appreciation of the commercial, thereby suppressing the direct negative effect a complete spelling out of the message had on appreciation. Without taking the suppressor into account, the effect of $\mathrm{X}$ on $\mathrm{Y}$ (the total effect $\mathrm{c}$ ) is estimated too low. If the suppressor is taken into account, the effect of $\mathrm{X}$ on $\mathrm{Y}$ (the direct effect $c^{\prime}$ ) should be higher than the total effect $c$. This implies that the polarity of ab (a multiplied by b) is different from c. It is therefore suppression and not mediation that applies here.

\section{ACKNOWLEDGMENTS}

The authors thank global market research agency MetrixLab for providing the TV commercials and the consumer responses to these TV commercials. The coding checklist can be obtained from the first author.

\section{REFERENCES}

Ang, Swee Hoon, and Elison A.C. Lim (2006), "The Influence of Metaphors and Product Type on Brand Personality, Perceptions, and Attitudes," Journal of Advertising, 35 (2), 39-53.
Barthes, Roland (1977), Image Music Text, New York: Hill and Wang.

- (1985), "The Rhetoric of the Image," in The Responsibility of Forms: Critical Essays on Music, Art, and Representation, New York: Hill and Wang, 21-40.

Burgers, Christian, Margot van Mulken, and Peter Jan Schellens (2012), "Type of Evaluation and Marking of Irony: The Role of Perceived Complexity and Comprehension," Journal of Pragmatics, 44, 231-242.

Corbett, Edward P.J., and Robert J. Connors (1999), Classical Rhetoric for the Modern Student (4th ed.), New York: Oxford University Press.

Dingena, Marian (1994), The Creation of Meaning in Advertising: Interactions of Figurative Advertising and Individual Differences in Processing Styles, Amsterdam: Thesis Publishers.

Eisend, Martin (2011), "How Humor in Advertising Works: A Meta-Analytic Test of Alternative Models," Marketing Letters, 22, 115-32.

Forceville, Charles (1996), Pictorial Metaphor in Advertising, London: Routledge.

Grice, H. Paul (1975), "Logic and Conversation," in Speech Acts, Peter Cole and Jerry L. Morgan, eds., New York: Academic Press, 41-58.

Hoeken, Hans, Corine van den Brandt, Rogier Crijns, Nuria Domínguez, Berna Hendriks, Brigitte Planken, and Marianne Starren (2003), "International Advertising in Western Europe: Should Differences in Uncertainty Avoidance be Considered when Advertising in Belgium, France, the Netherlands and Spain?," Journal of Business Communication, 40 (3), 195-218.

- Marianne Starren, Catherine Nickerson, Rogier Crijns, and Corine van den Brandt (2007), "Is it Necessary to Adapt Advertising Appeals for National Audiences in Western Europe?," Journal of Marketing Communications, 13 (1), 19-38.

_ Form to Stimulate Conversations: The Case of Tropes," Communication Theory, 19 (1), 49-65.

Hornikx, Jos, and Daniel J. O’Keefe (2009), “Adapting Consumer Advertising Appeals to Cultural Values: A Meta-Analytic Review of Effects on Persuasiveness and Ad Liking," in Communication Yearbook 33, Christina S. Beck, ed., New York: Erlbaum, 38-71.

Jeong, Se-Hoon (2008), "Visual Metaphor in Advertising: Is the Persuasive Effect Attributable to Visual Argumentation or Metaphorical Rhetoric?," Journal of Marketing Communications, 14 (1), 59-73.

Kardes, Frank R. (1988), "Spontaneous Inference Processes in Advertising: the Effects of Conclusion Omission and Involvement on Persuasion," Journal of Consumer Research, 15, 225-33.

- John Kim, and Jeen-Su Lim (1994), "Moderating Effects of Prior Knowledge on the Perceived Diagnosticity of Beliefs Derived from Implicit versus Explicit Product Claims," Journal of Business Research, 29 (3), 219-24.

Ketelaar, Paul E., and Marnix S. van Gisbergen (2006), "Openness in Advertising: Occurrence and Effects of Open Advertisements in Magazines," doctoral dissertation, Radboud University.

$\longrightarrow$, Jan A.M. Bosman, and Johannes Beentjes (2010), "The Effects of Openness on Attitude Toward the Ad, Attitude Toward the Brand, and Brand Beliefs in Dutch Magazine Ads," Journal of Current Issues and Research in Advertising, 32 (2), 71-85.

Lee, Yih Hwai, and Charlotte Mason (1999), "Responses to Information Incongruency in Advertising: The Role of Expectancy, Relevancy, and Humor," Journal of Consumer Research, 26, 156-69.

le Pair, Rob, and Margot van Mulken (2010), "Effects of Verbal Anchoring in Visual Metaphors on Perceived Complexity and Appreciation of Advertisements," in Topical Issues of Advertising: Theory and Practise, Alexander V. Prokhorov, ed., Tambov: TSU, 26-36.

Maes, Alfons, and Joost Schilperoord (2009), "Schemes and Tropes in Visual Communication: the Case of Object Grouping in Advertisements," in Discourse Off Course, Jan Renkema, ed., Amsterdam: Benjamins, 65-76.

McQuarrie, Edward F., and David G. Mick (1996), "Figures of Rhetoric in Advertising Language," Journal of Consumer Research, 22, 424-38. 
_ , and (2003a), "The Contribution of Semiotic and Rhetorical Perspectives to the Explanation of Visual Persuasion in Advertising," in Persuasive Imagery: A Consumer Response Perspective, Linda M. Scott and Rajeev Batra, eds., Mahwah, NJ: Erlbaum, 191-221.

, and (2003b), "Visual and Verbal Rhetorical Figures under Directed Processing Versus Incidental Exposure to Advertising," Journal of Consumer Research, 29, 579-87.

Messaris, Paul (1997), Visual Persuasion: The Role of Images in Advertising, Thousand Oaks, CA: Sage.

Nisbett, Richard E. (2003), The Geography of Thought: How Asians and Westerners Think Differently-and Why, London: Nicholas Brealey Publishing.

Phillips, Barbara J. (2000), "The Impact of Verbal Anchoring on Consumer Response to Image Ads," Journal of Advertising, 29 (1), 15-24.

, and Edward F. McQuarrie (2002), "The Development, Change, and Transformation of Rhetorical Style in Magazine Advertisements 1954 1999," Journal of Advertising, 31 (4), 1-13.

- and (2004), "Beyond Visual Metaphor: A New Typology of Visual Rhetoric in Advertising," Marketing Theory, 4 (1/2), 113-36.

Preacher, Kristopher J., and Andrew F. Hayes (2008), "Contemporary Approaches to Assessing Mediation in Communication Research," in The Sage Sourcebook of Advanced Data Analysis Methods for Communication Research, Andrew F. Hayes, Michael D. Slater, and Leslie B. Snyder, eds., Thousand Oaks, CA: Sage, 13-54.

Sawyer, Alan G., and Daniel J. Howard (1991), "Effects of Omitting Conclusions in Advertisements to Involved and Uninvolved Audiences," Journal of Marketing Research, 28 (November), 467-74.

Shrout, Patrick E., and Niall Bolger (2002), "Mediation in Experimental and Nonexperimental Studies: New Procedures and Recommendations," Psychological Methods, 7 (4), 422-45.

Sperber, Dan, and Deirdre Wilson (1995), Relevance: Communication and Cognition (2nd ed.), Oxford: Blackwell.
Spooren, Wilbert, and Liesbeth Degand (2010), "Coding Coherence Relations: Reliability and Validity," Corpus Linguistics and Linguistic Theory, 6 (2), 241-66.

Stewart, David W., and David H. Furse (2000), "Analysis of the Impact of Executional Factors on Advertising Performance," Journal of Advertising Research, 40 (6), 85-88.

van Enschot, Renske, Cyrella Beckers, and Margot van Mulken (2010), "Rhetorical Figures in TV Commercials: The Occurrence of Schemes and Tropes and Their Effects on Commercial Likeability," Information Design Journal, 18 (2), 138-47.

- Hans Hoeken, and Margot van Mulken (2008), "Rhetoric in Advertising: Attitudes towards Verbo-Pictorial Rhetorical Figures," Information Design Journal, 16 (1), 35-45.

van Hooft, Andreu, Margot van Mulken, and Ulrike Nederstigt (2011), "Cultural Differences? Visual Metaphor in Advertising: Comprehension and Tolerance of Ambiguity in Four European Countries," In Advances in Advertising Research (Vol. 4), Sara Rosengren, Micael Dahlén, and Shintaro Okazaki, eds., Wiesbaden: Springer, 351-64.

van Mulken, Margot, Renske van Enschot-Van Dijk, and Hans Hoeken (2005), "Puns, Relevance and Appreciation in Advertisements," Journal of Pragmatics, 37, 707-21.

- Rob le Pair, and Charles Forceville (2010), "The Impact of Perceived Complexity, Deviation, and Comprehension on the Appreciation of Visual Metaphor in Advertising across Three European Countries," Journal of Pragmatics, 42, 3418-30.

Zarantonello, Lia, Kamel Jedidi, and Bernd H. Schmitt (2013), "Functional and Experiential Routes to Persuasion: An Analysis of Advertising in Emerging versus Developed Markets," International Journal of Research in Marketing, 30 (1), 46-56.

Zhou, Nan, and Russell W. Belk (2004), "Chinese Consumer Readings of Global and Local Advertising Appeals," Journal of Advertising, 33 (3), 63-76. 


\section{APPENDIX}

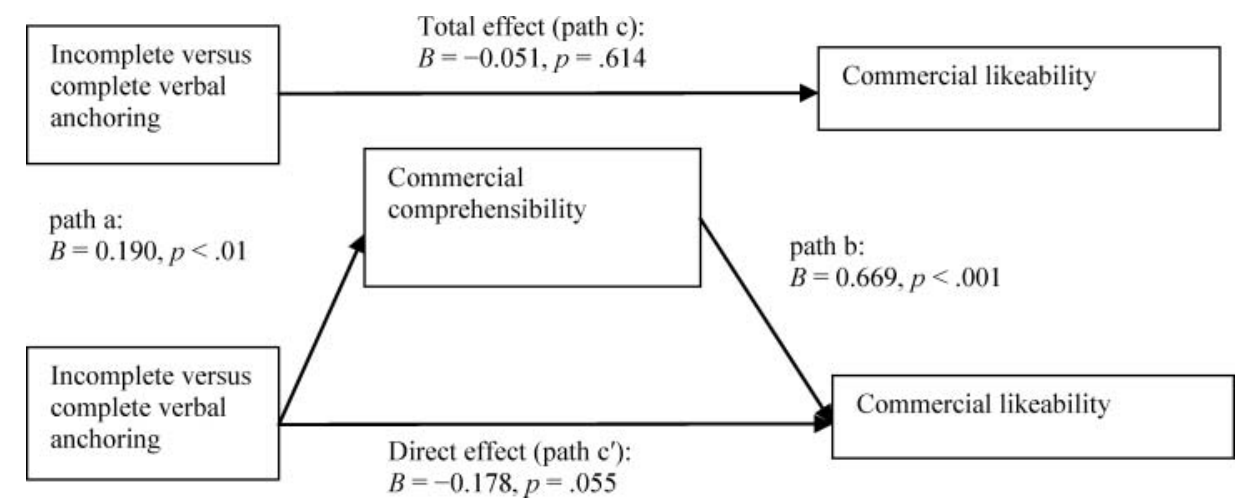

FIG. A1. Suppression effect for the extent of verbal anchoring via perceived commercial comprehensibility on commercial likeability.

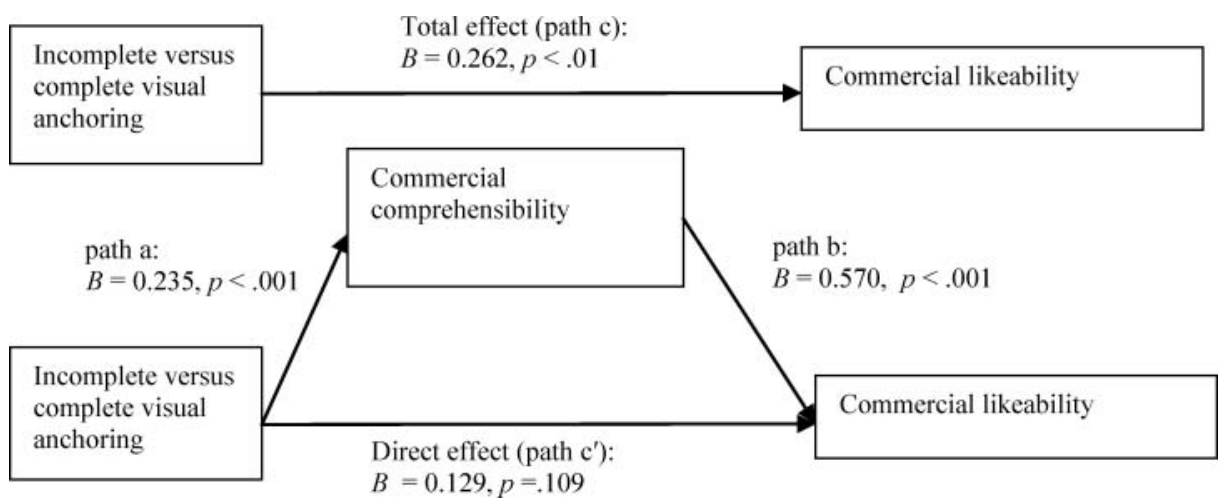

FIG. A2. Mediation effect for the extent of visual anchoring via perceived commercial comprehensibility on commercial likeability. 